\title{
On Some New or Little Known Plants from Northern China.
}

$\mathrm{By}$

Yoshitada Yabe.

1. Panicum trypheron Schult. Mant. II. 24t; Hoor. f. Fl. Br. Ind. VII. p. 47; Rendle in Journ. Linn. Soc. XXXVI. p. 333.

Hab. North China: dry sandy plain of Nan-haitze near Peking (Sept. 1906. fl. fr.); Manchuria: Moukden (21. Aug. 1914 fr.).

This south Asiatic species hitherto only known from Tientsin.

2. Deschampsia chinensis M.

Root firbrous. Culm tufted, smooth, very slender, capillary, $20-25 \mathrm{~cm}$. long. Leaves almost all radical, 3-5 cm. long, very narrow, capillary, straight; ligule short, truncate. Panicle spreading, sub-nutant, trichotomous; branches few, slender, capillary, scabrous; branchlets short alternate. Spikelets $6 \mathrm{~mm}$. long, 2-flowered, purple. Glume I smallest, $2.5-3 \mathrm{~mm}$. long, ovate-lanceolate, acute, 1-nerved; gl. II $3.5 \mathrm{~mm}$. long, 3-nerved, pilose at the base; gl. III 5-6 $\mathrm{mm}$. long, acuminate, pointed to the awn, 5-nerved, ovate, purple striped at the tip. Palea 4.5 mm. long, narrow ovate, 2 keeled; keel setose. Anthers small.

Very closely related to Aira atropurpurea and D. flexuosa, but easily distinguishable by rather small spikes and gl. III acute or acuminate, not truncated.

Hab. North China: near the summit of Mt. Siaowutaishan, about 3000 m. alt. (31. Jul. 1906 fl).

3. Pappophorum brachystachyum Jaub. et Spach. "Ill. Pl. Orient. IV. t. 824"; STEUd. Syn. Gram. p. 200. Enneapogon 
brachystachyus StapF. Rendle in Journ. Linn. Soc. XXXVI. p. 407.

Hab. North China: Peking, on the dry wall of the city (Aug. flow.), very common; Eastern Mongolia: Shih-chia-tzu -1家子 (S. Hashimoto 29. Aug. 1910).

4. Melica Gmelini Turcz. Steud. Syn. Gram. p. 289 ; RgL. Tent. Fl. Ussur. p. 169; Maxim. Prim. Fl. Amur. p. 322; Franch. Pl. David. I. p. 336; Komarov, Fl. Mansh. I. p. 296; Rendre in Journ. Linn.' Soc. XXXVI. p. 418.

Hab. North China: at the summit of Mt. Pohuashan alt. $2500 \mathrm{~m}$ ( Jul. 1905); alpine region of Mt. Siao-wutaishan (Jul. 1906) ; Mt. Wutaishan (Jul. 1907).

Northern Manchuria: Keelin, along the river Soongari (Sunghua-chiang). (June 1914. T. KACHI coll).

5. Bromus inermis Leyss. Fl. Hall. p. 16 ; Steud. Syn. Gram. p. 321; StrapF in Hook. f. Fl. Br. Ind. VII. p. 357 ; Aschers. u. Graebn. Syn. Mitteleur. F1. II. 1. p. 589.

Hab. Shan-hsi: Eastern peak of Mt. Wutaishan (Jul. 1907. and Y. Nagai Jul. 1908).

6. Stipa mongholica Turcz. Steud. Syn. Gr.p. 132; Hook. f. Fl. Br. Ind. p. 229; Rendle in Journ. Linn. Soc. XXXVI. p. 382 ; Ptilagrostis mongolica Griseb. in Ledeb. Fl. Ross. IV. p. 447; Turcz. Fl. Baic. Dah. II. 1. p, 302.

Hab. Prov. Chihli: at the top of Mt. Siao-wutai (jul. 1906); Shanhsi: Mt. Wutaishan (Jul. 1907 fl.).

7. Carex (Hymenochlaenae) capillaris L. var. pohuasharensis M. Rhizome dense caespitose. Stem 20-35 cm. long, strict, filiform, obtuse-angled, smooth, leafy below. Leaves much shorter than the culm, $10-25 \mathrm{~cm}$. long, $3 \mathrm{~mm}$. broad, flat, often prolonged and forming scabrous acumen. Vagina brownish at base, margin more or less fibrous. Spikelets 4-5; terminal one staminate or sometimes gynaecandrous, linear, 7-10 mm. long, erect or nutating; peduncles slender scabrous. Lateral three or four spiklets $q$ oblong 8-13 flowered; the lowest long pedunculate 15-18 mm. long, $3 \mathrm{~mm}$. diam. lax-flowered, subcernuous; bracts long, foliaceous. Glumes $\sigma^{\top}$ obovate-oblong, rotundate, margin hyaline; glumes $q$ obovate $2 \mathrm{~mm}$. long, apex rounded 
or obtuse or shortly acuminate, green-keeled, margin broad, scabrous. Rachis scaberulous. Utricles $3 \mathrm{~mm}$. long, ovateelliptical, membranaceous, brown, nerveless, apex produced into a scabrous beak. Nuts trigonous, style-base swollen, stigma 3 .

Very near to "forma 2 major" of KuEkENTHaL (Pflanzereich IV. 20. p. 590).

Hab. Chihli: among grasses, near the top of Mt. Po-huashan (Jul. 1908); Mt. Siao-wutaishan (Jul. 1906).

8. Listera Bungeana $\mathrm{M}$.

Root slender, fibrous. Stem erect, slender, 2-foliate, about $20 \mathrm{~cm}$. high. Leaves opposite, sessile, orbiculate-ovate, $11 / 2 \mathrm{~cm}$. long, $1.8 \mathrm{~cm}$. broad, apex obtuse, membranaceous. Raceme pubescent 5-7 flowered, flowers light brown. Dorsal sepal ovate-obtuse, $3.5 \mathrm{~mm}$. long, erect; lateral sepals $2.5 \mathrm{~mm}$. long, broad lanceolate, acute. Petals subequal; labellum oblong. 6 $\mathrm{mm}$. long, $2.5 \mathrm{~mm}$. broad, 2-lobed, lobes entire obtuse, median line thickened. Bracts ovate acute or obtuse, $3 \mathrm{~mm}$. long; pedicels slender, shorter than the bracts.

Hab. Chihli: near the summit of Mt. Pohuashan, about 2500 m. alt. (Jul. 1905).

Very closely related to L. Eschscholziana Cham.

9. Pteroceltis Tatarinowi Maxim. Mél. Biol. IX. p. $26 \mathrm{cum}$. fig., F1. As. Or. Fragm. p. 53 ; Benth. et Hook. Gen. Pl. III. p. 354; Forbes et HemsL. in Journ. Linn. Soc. XXVI. p. 451; Schneider, Ill. Laubholzk. I. p. 227.

Hab. Shan-tung: Lung tung near Chi-nanfu (Fukuyama 22. May 1915 fr.).

It seems rather good to exclude this species from Peking flora. There is no wild plant.

10. Holosteum umbellatum L. Sp. Pl. ed. 1. p. 130; DC. Prodr. I. p. 393; FenzL. in Ledeb. Fl. Ross. I. p. 373; EdGeworth et Hook. in Hook. f. Fl. Br. Ind. I. p. 227.

Hab. Chihli: near the summit of Mt. Siaowutaishan (Jul. 1906 fr.) New to Chinese flora.

11. Clematis Matsumurana m.

Scandent. Leaves long-petiolate, pinnate; segments 3-5, oblong-ovate, obtuse, $5-5.5 \mathrm{~cm}$. long, $1-2.5 \mathrm{~cm}$. broad, coria- 
ceous, glabrous, glaucous, more or less shining above, very distinctly reticulated; common petioles $10 \mathrm{~cm}$. long, petiolules 2 $\mathrm{cm}$. long. Young stem and peduncles hirsute, at length glabrous. Inflorescence axillary, paniculate. Flowers erect, spreading, 2 $\mathrm{cm}$. or more in diam. Sepals white, blackened when dried, 12$18 \mathrm{~mm}$. long, $6 \mathrm{~mm}$. broad, ellipsoid, truncate emarginate, subglabrous. Stamens numerous, shorter than the sepals, $6 \mathrm{~mm}$. long, anthers $2 \mathrm{~mm}$. long, oblong, filaments filiform glabrous.

Hab. Chihli: in a village Chan-tsao near Fan-shan-hsien (Jul. 1905 fl.)

12. Corydalis Bungeana Turcz. Bull. Soc. Not. Mosc. XIX. 1. p. 62; Hance in Journ. Bot. 1875. p. 130; Debeaux, Fl. Shangh. p. 16 ; Franch. Pl. Davd. I. p. 29; Forbes et Hemsi. in Journ. Linn. Soc. XXIII. p. 36 ; Maxim. Pl. Chin. Potan. p. 50; Palibin, Act. Hort. Petr. XIV. p. 108; Komarov, Fl. Mansh. II. p. 348 .

Hab. Shan-tung: Lung-tung near Chinanfu (Fukurama May 1915).

13. Sisymbrium Maximowiczi Palıbin. Consp. Fl. Kor. I. P. 18, t. 3; Matsumura in Tokyo. Bot. Mag. XVI. p. 17 ; NakaI, F1. Kor. I. p. 58.

Hab. Chihli: Hills near Shanhaikwan (Aug. 1907 fl. fr.) ; Manchuria: Mt. Laotieh-shan in Port Arthur (Aug. 1914).

14. Bunias tcheliensis Debeaux, Fl. Tientsin. p. 12; Forbes et Hemsl. in Journ. Linn. Soc. XXIII, p 49.

Hab. Chihli: Tientsin (G. Otsu May 1908); Eastern Mongolia: Tiaonan-fu (Mryoshi May 1914; S. Hashmoto Aug. 1910).

15. Chamœrhodos grandiflora BGE. in Ledeb. Fl. Alt. I. p. 431; F1. Ross. II. p. 34; Maxim. Ind. Fl. Mongol. p. 481; Palibin in Act. Hort. Petr. XIV. p. 117.

Hab. Chihli: Mt. Siao-wutaishan (Jul. 1906). Manchuria: Tailien (Dalny) (K. Kondo Jul. 1914).

16. Chamœrhodos trifida Ledeb. Fl. Ross. II. p. 34. C. altaica var. Bunge. "Enum. Alt. p. 19."

Eastern Mongolia, east of Jehol without special loc. (R. TORII). 